\title{
Erratum to: Venous Thromboembolism Prevention and Prophylaxis
}

Matteo Manici, Giacomo Alemanno, and Margherita I. Nuzzaco

Erratum to:

Chapter 13 in: I. Comisso et al., Nursing in Critical Care Setting, https://doi.org/10.1007/978-3-319-50559-6

The author name was misspelled as Magherita I. Nuzzacco and it has now been corrected as Margherita I. Nuzzaco.

The updated online version of the original chapter can be found at https://doi.org/10.1007/978-3-319-50559-6_13 